\title{
FORMAL MODULI OF MODULES OVER LOCAL $k$-ALGEBRAS
}

\author{
ALLAN ADLER AND PRADEEP SHUKLA
}

\begin{abstract}
We determine explicitly the formal moduli space of certain complete topological modules over a topologically finitely generated local $k$-algebra $R$, not necessarily commutative, where $k$ is a field. The class of topological modules we consider include all those of finite rank over $k$ and some of infinite rank as well, namely those with a Schauder basis in the sense of $\S 1$. This generalizes the results of [Sh], where the result was obtained in a different way in case the ring $R$ is the completion of the local ring of a plane curve singularity and the module is $k^{n}$. Along the way, we determine the ring of infinite matrices which correspond to the endomorphisms of the modules with Schauder bases. We also introduce functions called "growth functions" to handle explicit epsilonics involving the convergence of formal power series in noncommuting variables evaluated at endomorphisms of our modules. The description of the moduli space involves the study of a ring of infinite series involving possibly infinitely many variables and which is different from the ring of power series in these variables in either the wide or the narrow sense. Our approach is beyond the methods of [Sch] which were used in [Sh] and is more conceptual.
\end{abstract}

\section{INTRODUCTION}

In his thesis [Sh], the second author of this paper proved the following result.

(0.1) Theorem. The moduli space $\mathscr{M}(2, d)$ of semistable torsion-free sheaves of rank 2 and degree $d$ over an integral curve is reduced provided that the curve has only ordinary cusps and nodes as singularities.

This result had been proved by D'Souza [D] in the case of torsion-free sheaves of rank 1, while Seshadri [Sesh] initiated the study of moduli of torsion-free sheaves of higher rank. The proof of Theorem $(0.1)$ depends on the following solution (cf. [Sh, Theorem 1.13, p. 20]) to a formal moduli problem.

(0.2) Theorem. Let $R$ be the completion of the local ring of a plane curve singularity and let $k$ be its residue field. Let $F$ denote the deformation functor of the $R$-module $k^{n}=k \oplus k \oplus \cdots \oplus k$ with a fixed basis. Explicitly, $F$ associates to

Received by the editors March 18, 1990 and, in revised form, January 10, 1991.

1980 Mathematics Subject Classification (1985 Revision). Primary 11S20, 13D10, 13J05, 13J10, 14B07, 14B10, 14B12, 15A33, 20M35, 20M50.

Key words and phrases. Formal moduli space, artin local ring, prorepresentable functor, Schauder basis, formal power series in noncommuting variables, free monoid, deformations of Galois representations, free topological $A$-module, matrix of a continuous endomorphism, growth function, order of a matrix, admissible homomorphism, formal power series in the narrow sense, formal power series in the wide sense, universal deformation ring. 
each artin local $k$-algebra $A$ with residue field $k$ the set of all $A$-free $R$-modules with an A-basis which restrict to the given module and its basis after tensoring with $k$. If $f(x, y)=0$ is the equation defining the curve singularity then $F$ is represented by the complete local ring

$$
\bar{A}=k\left[\left[x_{i j}, y_{i j} \mid 1 \leq i, j \leq n\right]\right] / I,
$$

where $I$ is the ideal generated by the entries of the matrices $[\widehat{X}, \widehat{Y}]=\widehat{X} \hat{Y}-\widehat{Y} \hat{X}$ and $f(\widehat{X}, \widehat{Y})$ and where $\widehat{X}=\left(x_{i j}\right)$ and $\widehat{Y}=\left(y_{i j}\right)$.

Note that although the expression $f(\widehat{X}, \widehat{Y})$ is ambiguous, its value modulo the entries of $[\widehat{X}, \widehat{Y}]$ is well defined. This theorem generalized one of Seshadri's results [Sesh] which handled the case $f(x, y)=x y$.

The second author obtained this theorem in [Sh] using the methods of Schlessinger [Sch] and the proof did not easily lend itself to the study of modules other than $k^{n}$. Subsequent discussions with the first author led to a more general approach which did not depend on Schlessinger's methods. It soon became apparent that the setting for such results goes far beyond the requirements of the original moduli problem for torsion-free sheaves on curves and is of independent interest. More precisely, we have proved the following result (cf. Theorem 5.6 of this paper).

(0.3) Theorem. Let $R$ be the image of the $k$-algebra of formal power series in a finite number of noncommuting variables $x_{1}, \ldots, x_{n}$ with coefficients in $k$ under a continuous homomorphism with kernel $I$. Let $M$ be a complete separated topological $R$-module, not necessarily finitely generated but having a $S c h a u d e r$ basis $\beta$ in the sense of Definition 5.5. If $x_{i}$ acts on $M$ via the matrix $P_{i}$ with respect to the $S$ chauder basis $\beta$ then the formal moduli space $\mathscr{M}$ of $M$ is the formal spectrum of the complete local ring

$$
\bar{A} / \mathscr{I}=\frac{k\left[\left[\left[\widehat{X}_{1}, \ldots, \widehat{X}_{n}\right]\right]\right]}{\left\langle f\left(\widehat{X}_{1}+P_{1}, \ldots, \widehat{X}_{n}+P_{n}\right) \mid f \in I\right\rangle}
$$

where $\widehat{X}_{1}, \ldots, \widehat{X}_{n}$ are $\beta \times \beta$ matrices of indeterminates and $k\left[\left[\left[\widehat{X}_{1}, \ldots, \widehat{X}_{n}\right]\right]\right]$ is a certain ring of formal series in the entries of the matrices $\widehat{X}_{1}, \ldots, \widehat{X}_{n}$.

The precise definition of the $k$-algebra $\bar{A}=k\left[\left[\left[\widehat{X}_{1}, \ldots, \widehat{X}_{n}\right]\right]\right]$ is given in $\S 4$. In general, $\bar{A}$ is not noetherian and its topology is in general not the $\mathscr{M}_{\bar{A}}$ adic topology, where $\mathscr{M}_{\bar{A}}$ is the maximal ideal of $\bar{A}$. Consequently these results are beyond the scope of the methods of Schlessinger's paper [Sch]. However, in case $M$ has finite rank over $k$, the $k$-algebra $\bar{A}$ is noetherian.

It is quite easy to prove that the formal spectrum of the right-hand side of (0.4) is the moduli space once one has succeeded in giving all of the definitions of the terms in the right-hand side and proving that they make sense. That is what constitutes the bulk of this paper. The description of elements of $R$ acting on $M$ by matrices involves choosing a basis, but since we wish to substitute these matrices in formal power series, we have to consider questions of convergence. Therefore, we cannot use Hamel bases for $M$ but instead introduce an appropriate topological notion of basis which we call a Schauder basis (cf. $\S 1$ and Definition 5.5), in imitation of the terminology used in functional analysis.

In $\S 1$ we discuss linear algebra in terms of Schauder bases and in Proposition 1.2 characterize those matrices which correspond to continuous linear 
operators. Such a characterization is necessary to prove that expressions such as $f\left(\widehat{X}_{1}+P_{1}, \ldots, \widehat{X}_{n}+P_{n}\right)$ actually correspond to continuous operators. Even more fundamental than the continuity of such expressions is their existence: we have to prove that the series converge. For this, we introduce a tool called a growth function which is used for explicit epsilonics. Growth functions are discussed in $\S 2$. In $\S 3$, we use growth functions to prove the convergence of certain power series involving matrices with entries in an artin local $k$-algebra. These are power series in noncommuting variables evaluated at matrix arguments. The free monoid on these noncommuting variables is a Schauder basis for the $k$ algebra of formal power series in the noncommuting variables with coefficients in $k$ and the module $M$ can be viewed as a module of the free monoid. This is the point of view adopted in $\S 4$, where we first determine the moduli space $\mathscr{M}$ in case the ideal $I$ is zero, under the guise of determining the deformation functor for an admissible representation of the free monoid. It turns out that the algebra $\bar{A}$, which we define in $\S 4$, is precisely the prorepresenting object for the deformation functor on the category of artin local $k$-algebras. In $\S 5$, we show that the modules which arise as deformations actually have Schauder bases and use this result to prove the result $(0.3)$ in the form of Theorem 5.6.

The solution of the general formal moduli problem makes it possible, in principle, to pursue further the study of the moduli space $\mathscr{M}(r, d)$ of semistable torsion-free sheaves of rank $r$ and degree $d$ over a curve with more general singularities. In particular, since torsion-free modules over a simple curve singularity were completely classified by Geuel and Knörrer [G-K], it may be possible to study the moduli space $\mathscr{M}(r, d)$ when the curve has simple singularities. Furthermore, specializing to the case $r=1$ amounts to studying the compactified Picard variety of a singular curve, which has been carried out for special curves by several authors, e.g. [A-I-K, D, G, O-S].

Another possible direction for further work is motivated by Mazur's recent paper $[\mathrm{M}]$ on deformations of representations of Galois groups. According to Serre's book [Ser], if we denote by $F_{n}$ the free pro- $p$ group on $n$ generators $\gamma_{1}, \ldots, \gamma_{n}$ then the complete group ring $\mathbf{Z}_{p}\left[\left[F_{n}\right]\right]$ of $F_{n}$ over the ring $\mathbf{Z}_{p}$ of $p$-adic integers is isomorphic to the ring of formal power series in noncommuting variables $x_{1}, \ldots, x_{n}$ with coefficients in $\mathbf{Z}_{p}$. If we tensor with a field $k$ of characteristic $p$ then we conclude that the complete group algebra $k\left[\left[F_{n}\right]\right]$ of $F_{n}$ over $k$ is isomorphic to the $k$-algebra of formal power series in $x_{1}, \ldots, x_{n}$ with coefficients in $k$. Therefore, if $k$ has characteristic $p$, the study of formal moduli we have undertaken in relation to the study of torsion-free sheaves is contained in the study of deformations of representations of the free pro- $p$ group $F_{n}$. This suggests that one should study deformations of representations of arbitrary profinite groups, including deformations to characteristic zero. It seems quite reasonable to expect that Theorem $(0.3)$ should extend to this situation. One can then consider the relation of our moduli spaces to the ones Mazur obtains.

\section{Topological Linear algebra}

Let $A$ be a topological ring and let $S$ be a topological space with the discrete topology. If $U$ is a subset of $A$, we will denote by $\mathscr{C}(S, U)$ the set of all functions from $S$ to $U$. Furthermore, if $S^{*}=S \cup\{\infty\}$ is the one point 
compactification of $S$ and if $U$ contains 0 , then we will denote by $\mathscr{C}_{0}(S, U)$ the set of those functions in $\mathscr{C}(S, U)$ which are restrictions to $S$ of continuous functions $f: S^{*} \rightarrow U$ such that $f(\infty)=0$. We will refer to the elements of $\mathscr{C}_{0}(S, U)$ as continuous functions from $S$ to $U$ vanishing at $\infty$.

We regard $\mathscr{C}(S, A)$ as a topological $A$-module by giving it the topology of uniform convergence. With respect to the relative topology $\mathscr{C}_{0}(S, A)$ is also a topological $A$-module. If $U$ and $V$ are neighborhoods of 0 in $A$ and if $U \subseteq V$ then the set $\mathscr{C}_{0}(S, U)$ can be canonically regarded as a subset of the set $\mathscr{C}_{0}(S, V)$. It is easy to see that the sets of the form $\mathscr{C}_{0}(S, U)$, where $U$ is an open neighborhood of 0 in $A$, form a base for the neighborhoods of 0 in $\mathscr{C}_{0}(S, A)$. We note that since $S$ has the discrete topology, $\mathscr{C}_{0}(S, A)$ consists of all functions $f: S \rightarrow A$ with finite support and that $\mathscr{C}_{0}(S, U)$ is the direct sum of copies of $A$ indexed by $S$.

Let $\beta=\bigvee \beta_{i}$ be a disjoint union of discrete topological spaces $\beta_{i}$, where $i$ runs over all nonnegative integers. We denote by $\mathscr{S}(A ; \beta)$ the $A$-module $\prod \mathscr{C}_{0}\left(\beta_{i}, A\right)$ and give it the product topology. Thus an element of $\mathscr{S}(A ; \beta)$ may be regarded as a function from $\beta$ to $A$ whose restriction $f_{i}$ to $\beta_{i}$ vanishes at $\infty$ for all $i$. We will call $\mathscr{S}(A ; \beta)$ the free topological $A$-module with $S c h a u d e r$ basis $\beta$. The reader can find the motivation for this definition by referring to Lemmas 5.3 and 5.4.

If $M$ is a topological $A$-module, we will denote by $\operatorname{End}_{A}^{c}(M)$ the ring of continuous $A$-endomorphisms of $M$. Our goal in this section is to find an explicit description of $\operatorname{End}_{A}^{c}(\mathscr{S}(A ; \beta))$ as a ring of matrices.

First we introduce some terminology. Let $X$ and $Y$ be nonempty sets. An $X \times Y$ matrix $f$ with entries in a set $R$ is a function from $X \times Y$ to $R$. The value $f(x, y)$ of $f$ at an element $(x, y)$ of $X \times Y$ is called the $(x, y)$ th entry of the matrix $f$.

Let $S=\left\{a_{\lambda} \mid \lambda \in \Lambda\right\}$ be a subset of $A$ indexed by a set $\Lambda$. Clearly the set of finite sums of elements of $S$ forms a net indexed by finite subsets of $\Lambda$. If this net converges we will denote its limit by $\sum_{\lambda \in \Lambda} a_{\lambda}$.

(1.1) Definition. Let $T$ be an $A$-endomorphism of $\mathscr{S}(A ; \beta)$, continuous or not. The $\beta \times \beta$ matrix $f_{T}$ given by

$$
f_{T}\left(b^{\prime}, b\right)=T(b)\left(b^{\prime}\right)
$$

is called the matrix of $T$ with respect to the Schauder basis $\beta$. If $T$ is the identity mapping from $\mathscr{S}(A ; \beta)$ to itself, we denote the matrix of $T$ by $\delta$. We then have

$$
\delta\left(b, b^{\prime}\right)= \begin{cases}1 & \text { if } b=b^{\prime} \\ 0 & \text { if } b \neq b^{\prime}\end{cases}
$$

(1.2) Proposition. If a matrix $f: \beta \times \beta \rightarrow A$ is the matrix of a continuous endomorphism of $\mathscr{S}(A ; \beta)$ then

(1) for every element $b$ of $\beta$ and every neighborhood $V$ of zero in $A$ there is a subset $U$ of $\beta$ such that

(i) $U \cap \beta_{i}$ is a cofinite subset of $\beta_{i}$ for every nonnegative integer $i$,

(ii) $f\left(b^{\prime}, b\right)$ belongs to $V$ for every element $b^{\prime}$ of $U$;

(2) if $n$ is a nonnegative integer and $V$ is a neighborhood of 0 in $A$ then there is a nonnegative integer $n_{0}$ such that for all $k \geq n_{0}$ the $\left(b^{\prime}, b\right)$ th entry $f\left(b^{\prime}, b\right)$ of the matrix $f$ lies in $V$ for all $\left(b^{\prime}, b\right)$ in $\beta_{n} \times \beta_{k}$. 
Conversely, let $f$ be a matrix satisfying (1) and (2). If $A$ is a complete linearly topologized ring (cf. [Gr]) then there is a unique continuous endomorphism of $\mathscr{S}(A ; \beta)$ whose matrix is $f$.

Proof. $(\Rightarrow)$ Let $T$ be a continuous $A$-endomorphism $\mathscr{S}(A ; \beta)$ and let $f$ be its matrix. Since $T(b)$ belongs to $\mathscr{S}(A ; \beta)$ and since

$$
T(b)=f_{T}\left(b^{\prime}, b\right)=f\left(b^{\prime}, b\right),
$$

for all elements $b^{\prime}$ of $\beta$, assertion (1) follows immediately from the definition of $\mathscr{S}(A ; \beta)$. By the continuity of $f$, for every nonnegative integer $n$ and every open neighborhood $V$ of 0 in $A$, there exists a nonnegative integer $n_{0}$ and open neighborhoods $\mathscr{C}_{0}\left(\beta_{i}, U_{i}\right)$ of 0 in $\mathscr{C}\left(\beta_{i}, A\right)$ for $1 \leq i \leq n_{0}$ such that for every element $u$ of

$$
\prod_{i=1}^{n_{0}} \mathscr{E}_{0}\left(\beta_{i}, U_{i}\right) \times \prod_{i>n_{0}} \mathscr{C}_{0}\left(\beta_{i}, A\right),
$$

the value $T(u)\left(b^{\prime}\right)$ lies in $V$ for all $b^{\prime}$ in $\beta_{n}$. In particular, for every integer $k \geq n_{0}$ and any element $b$ of $\beta_{k}$ the matrix entry $f\left(b^{\prime}, b\right)=f(b)\left(b^{\prime}\right)$ lies in $V$. This proves (2). Conversely, let $f: \beta \times \beta \rightarrow A$ be a matrix satisfying (1) and (2). Using (2) and the fact that $A$ is a complete linearly topologized ring, it is easy to see that for every element $b^{\prime}$ of $\beta$ and every element $s$ of $\mathscr{S}(A ; \beta)$ the series $\sum_{b \in \beta} f\left(b^{\prime}, b\right) \cdot s(b)$ converges to an element of $A$. We define an endomorphism $T$ of $S(A ; \beta)$ by

$$
T(s)\left(b^{\prime}\right)=\sum_{b \in \beta} f\left(b^{\prime}, b\right) \cdot s(b) .
$$

We claim that $T$ is continuous. Let $I$ be an open ideal of $A$. Consider the subbasic open set

$$
\mathscr{U}=\prod_{i=0}^{n-1} \mathscr{C}_{0}\left(\beta_{i}, A\right) \times \mathscr{C}_{0}\left(\beta_{n}, I\right) \times \prod_{i>n} \mathscr{C}_{0}\left(\beta_{i}, A\right)
$$

of $\mathscr{S}(A ; \beta)$. By (2), there is a nonnegative integer $n_{0}$ such that for every integer $k \geq n_{0}$ and for every element $\left(b^{\prime}, b\right)$ of $\beta_{n} \times \beta_{k}$ the matrix element $f\left(b^{\prime}, b\right)$ lies in the ideal $I$. Therefore for every element $s$ of the open set

$$
\mathscr{V}=\prod_{i=0}^{n_{0}-1} \mathscr{C}_{0}\left(\beta_{i}, I\right) \times \prod_{i \geq n_{0}} \mathscr{C}_{0}\left(\beta_{i}, A\right)
$$

of $\mathscr{S}(A ; \beta)$ every term of the series $\sum_{b \in \beta} f\left(b^{\prime}, b\right) \cdot s(b)$ is in $I$. In other words, $T(\mathscr{V})$ is contained in $\mathscr{U}$, which shows that $T$ is continuous. Finally, if $T^{\prime}$ is any other continuous endomorphism of $\mathscr{S}(A ; \beta)$ whose matrix is $f$ then

$$
T(b)\left(b^{\prime}\right)=T^{\prime}(b)\left(b^{\prime}\right)=f\left(b^{\prime}, b\right)
$$

for every $\left(b^{\prime}, b\right)$ in $\beta \times \beta$. Thus $T$ and $T^{\prime}$ agree on $b$ for all $b$ in $\beta$. Therefore $T$ and $T^{\prime}$ agree on the $A$-submodule of $\mathscr{S}(A ; \beta)$ generated by all $b$ with $b$ in $\beta$. Since this submodule is dense in $\mathscr{S}(A ; \beta)$ and since $T$ and $T^{\prime}$ are both continuous it follows that $T=T^{\prime}$. 
(1.3). We will denote by $M_{\beta}^{c}(A)$ the set of all $\beta \times \beta$ matrices with entries in $A$ which satisfy conditions (1) and (2) of Proposition (1.2). If $f_{1}$ and $f_{2}$ are $\beta \times \beta$ matrices with entries in $A$, their product in the sense of matrix multiplication is denoted $f_{1} * f_{2}$. Explicitly, we have for all $\left(b^{\prime}, b\right)$ in $\beta \times \beta$ that

$$
f_{1} * f_{2}\left(b^{\prime}, b\right)=\sum_{c \in \beta} f_{1}\left(b^{\prime}, c\right) \cdot f_{2}(c, b)
$$

provided that this sum converges in a suitable sense.

(1.4) Corollary. Let $A$ be a complete linearly topologized topological $k$-algebra. The set $M_{\beta}^{c}(A)$ of $\beta \times \beta$ matrices with entries in $A$ which satisfy conditions (1) and (2) of Proposition (1.2) forms a $k$-algebra with respect to matrix multiplication. If $A$ is commutative then $M_{\beta}^{c}(A)$ is actually an A-algebra. The map from $\operatorname{End}_{A}^{c}(\mathscr{S}(A ; \beta))$ to $M_{\beta}^{c}(A)$ which associates to a continuous endomorphism $T$ of $\mathscr{S}(A ; \beta)$ its matrix $f_{T}$ is an algebra isomorphism.

Proof. This is straightforward and is left to the reader.

\section{GROWTH FUNCTIONS}

We retain the assumptions and notation of the previous section. In this section, we introduce the notion of a growth function for a matrix.

(2.1) Definition. Let $\omega$ denote the set of nonnegative integers. A function $\mathscr{G}: \omega \rightarrow \omega$ is said to be increasing if $\mathscr{G}(i) \leq \mathscr{G}(i+1)$ for all $i$ in $\omega$. If $f: \beta \times \beta \rightarrow A$ is a matrix, an increasing function $\mathscr{G}: \omega \rightarrow \omega$ will be called a growth function for $f$ if we have $f\left(\beta_{i} \times \beta_{j}\right)=0$ for all nonnegative integers $i$ and $j$ such that $j \geq \mathscr{G}(i)$. The set of matrices $f: \beta \times \beta \rightarrow A$ such that $\mathscr{G}$ is a growth function for $f$ will be denoted $M_{\beta}(A ; \mathscr{G})$. If $n$ is a nonnegative integer and $\mathscr{G}: \omega \rightarrow \omega$ is an increasing function, we will denote by $\mathscr{G}_{n}$ the function from $\omega$ to $\omega$ defined by

$$
\mathscr{G}_{n}= \begin{cases}0 & \text { if } i<n, \\ \mathscr{G}(i-n) & \text { if } i \geq n .\end{cases}
$$

We denote by id the identity mapping of $\omega$. The growth function $\mathrm{id}_{n}$ is then defined by $\mathrm{id}_{n}(i)=\max (0, i-n)$.

(2.3) Lemma. Let $f_{1}$ and $f_{2}$ be $\beta \times \beta$ matrices with values in $A$ and let $\mathscr{G}_{1}$ and $\mathscr{G}_{2}$ be growth functions for $f_{1}$ and $f_{2}$ respectively. Then the function $\mathscr{G}: \omega \rightarrow \omega$ given by

$$
\mathscr{G}(i)= \begin{cases}0 & \text { if } \mathscr{G}_{1}(i)=0, \\ \mathscr{G}_{2}\left(\mathscr{G}_{1}(i)-1\right) & \text { if } \mathscr{G}_{1}(i)>0,\end{cases}
$$

is a growth function for $f_{1} * f_{2}$.

Proof. Let $f=f_{1} * f_{2}$ and let $\left(b^{\prime}, b\right)$ be an element of $\beta_{i} \times \beta_{j}$. Then

$$
\begin{aligned}
f\left(b^{\prime}, b\right) & =\sum_{\gamma \in \beta} f_{1}\left(b^{\prime}, \gamma\right) \cdot f_{2}(\gamma, b) \\
& =\sum \sum_{\gamma \in \beta_{k}} f_{1}\left(b^{\prime}, \gamma\right) \cdot f_{2}(\gamma, b),
\end{aligned}
$$


where the outer summation runs over all $k$ such that $0 \leq k<\mathscr{G}_{1}(i)$. In particular, the outer sum is empty if $\mathscr{G}_{1}(i)=0$. If $\mathscr{G}_{1}(i)>0$ then for each $k<\mathscr{G}_{1}(i)$ the inner sum is 0 in case $j \geq \mathscr{G}_{2}(k)$. Thus $f\left(b^{\prime}, b\right)=0$ if $\mathscr{G}_{1}(i)=0$ or if $\mathscr{G}_{1}(i)>0$ and

$$
j \geq \sup \left\{\mathscr{G}_{2}(k) \mid 0 \leq k \leq \mathscr{G}_{1}(i)\right\}=\mathscr{G}_{2}\left(\mathscr{G}_{1}(i)-1\right) .
$$

This proves that $\mathscr{G}$ is a growth function for $f$.

(2.4) Definition. We define the order of a matrix $f: \beta \times \beta \rightarrow A$ to be

$$
\inf \left\{i-j \mid f\left(\beta_{i} \times \beta_{j}\right) \neq\{0\}\right\} \text {. }
$$

We denote the order of $f$ by $\operatorname{ord}^{A}(f)$. We can have $\operatorname{ord}^{A}(f)= \pm \infty$. If $\operatorname{ord}^{A}(f)>-\infty$, we say that $f$ is bounded.

(2.5) Lemma. If $f: \beta \times \beta \rightarrow A$ is a matrix with ord $^{A}(f)=\nu \geq 0$ then the function $\operatorname{id}_{\nu-1}: i \mapsto \max (0, i-\nu+1)$ is a growth function for $f$.

Proof. If the restriction of $f$ to $\beta_{i} \times \beta_{j}$ is not zero, we have $\nu \leq i-j$ or, what is the same, $0 \leq j \leq i-\nu$. Therefore, $f\left(\beta_{i} \times \beta_{j}\right)=0$ if either $i \leq \nu$ or if $i \geq \nu$ and $j \geq i-\nu+1$. This proves the lemma.

(2.6) Corollary. Let $f_{1}: \beta \times \beta \rightarrow A$ be a bounded matrix with $\operatorname{ord}^{A}\left(f_{1}\right)=$ $\nu \geq 0$ and let $f_{2}: \beta \times \beta \rightarrow A$ be a matrix with growth function $\mathscr{G}$. Then $\mathscr{G}_{\nu}$ is a growth function for $f_{1} * f_{2}$ and $\mathrm{id}_{\nu} \circ \mathscr{G}$ is a growth function for $f_{2} * f_{1}$.

Proof. Let $f=f_{1} * f_{2}$. By Lemmas (2.3) and (2.5) the function $\mathscr{H}: \omega \rightarrow \omega$ given by

$$
\mathscr{H}(i)= \begin{cases}0 & \text { if } \mathrm{id}_{\nu-1}(i)=0, \\ \mathscr{G}\left(\mathrm{id}_{\nu}(i)-1\right) & \text { if } \mathrm{id}_{\nu-1}(i)>0,\end{cases}
$$

is a growth function for $f$. Since $\operatorname{id}_{\nu-1}(i)=0$ for $0 \leq i \leq \nu-1$ and $\operatorname{id}_{\nu-1}(i)=i-\nu+1$ for $i \geq \nu$ we have

$$
\mathscr{H}(i)= \begin{cases}0 & \text { if } i<\nu, \\ \mathscr{G}(i-\nu) & \text { if } i \geq \nu,\end{cases}
$$

which shows that $\mathscr{H}=i \mathscr{G} \nu$. To prove the second assertion of the corollary let $g=f_{2} * f_{1}$. By Lemmas (2.3) and (2.5) the function $\mathscr{J}: \omega \rightarrow \omega$ given by

$$
\mathscr{J}(i)= \begin{cases}0 & \text { if } \mathscr{G}(i)=0 \\ \operatorname{id}_{\nu-1}(\mathscr{G}(i)-1) & \text { if } \mathscr{G}(i)>0\end{cases}
$$

is a growth function for $g$. But $\mathscr{J}(i)=0$ for $\mathscr{G}(i) \leq \nu$ and if $\mathscr{J}(i)>\nu$ we have $\operatorname{id}_{\nu-1}(\mathscr{G}(i)-1)=\mathscr{G}(i)-\nu$. Hence $\mathscr{J}(i)=\mathrm{id}_{\nu}(\mathscr{G}(i))$ and therefore $\operatorname{id}_{\nu}(\mathscr{G}(i))$ is a growth function for $f_{2} * f_{1}$.

(2.7) Lemma. Let $\mathscr{G}: \omega \rightarrow \omega$ be an increasing function and let $\nu(1), \nu(2)$, $\ldots, \nu(n)$ be nonnegative integers. For $1 \leq j \leq n$ let $f_{j}: \beta \times \beta \rightarrow A$ be a matrix with growth function $\mathscr{G}_{\nu(n)}$. If $f=f_{1} * f_{2} * \cdots * f_{n}$ then the composition

$$
\left(\bigcirc_{i=2}^{n} \mathscr{G}_{\nu(i)+1}\right) \circ \mathscr{G}_{\nu(1)}=\mathscr{G}_{\nu(n)+1} \circ \cdots \circ \mathscr{G}_{\nu(2)+1} \circ \mathscr{G}_{\nu(1)}
$$

is a growth function for $f$.

Proof. For $1 \leq j \leq n$ define the function $\mathscr{H}^{(j)}: \omega \rightarrow \omega$ by

$$
\mathscr{H}^{(j)}(i)= \begin{cases}\mathscr{G}_{\nu(1)}(i) & \text { if } j=1, \\ 0 & \text { if } j>1 \text { and } \mathscr{H}^{(j-1)}(i)=0, \\ \mathscr{G}_{\nu(j)}\left(\mathscr{H}^{(j-1)}(i)-1\right) & \text { if } j>1 \text { and } \mathscr{H}^{(j-1)}(i)>0 .\end{cases}
$$


Then by Lemma (2.3) and by induction on $j$, the function $\mathscr{H}^{(j)}$ is a growth function for $f_{1} * \cdots * f_{j}$ for $1 \leq j \leq n$. From the definition of $\mathscr{H}^{(j)}$ we see that if $j>1$ then

$$
\mathscr{H}^{(j)}(i)= \begin{cases}0 & \text { if } \mathscr{H}^{(j-1)} \leq \nu(j), \\ \mathscr{G}\left(\mathscr{H}^{(j-1)}(i)-\nu(j)-1\right) & \text { if } \mathscr{H}^{(j-1)}>\nu(j) .\end{cases}
$$

Therefore if $j>1$ we have

$$
\mathscr{H}^{(j)}=\mathscr{G}_{\nu(j)+1} \circ \mathscr{H}^{(j-1)} .
$$

The lemma follows at once by induction.

(2.8) Lemma. Let $i$ be a nonnegative integer and let $n$ be a positive integer. Let $\mathscr{G}: \omega \rightarrow \omega$ be an increasing function. Let the integer $N$ be defined by

$$
N=\sum_{j=0}^{n-1} \mathscr{G}^{(j)}(i),
$$

where $\mathscr{G}^{(j)}$ denotes the $j$-fold composition of $\mathscr{G}$ with itself and where we adopt the convention that $\mathscr{G}^{(0)}(i)=i$. Let $\nu(1), \nu(2), \ldots, \nu(n)$ be nonnegative integers and for $1 \leq j \leq n$ let $f_{j}: \beta \times \beta \rightarrow A$ be a matrix with growth function $\mathscr{G}_{\nu(j)}$ and let

$$
f=f_{1} * f_{2} * \cdots * f_{n} .
$$

If $\sum_{j=1}^{n} \nu(j)>N-n+1$, then $f$ vanishes on $\beta_{i} \times \beta$.

Proof. Let $\mathscr{H}: \omega \rightarrow \omega$ be defined by

$$
\mathscr{H}=\mathscr{G}_{\nu(n)+1} \circ \mathscr{S}_{\nu(n-1)+1} \circ \cdots \circ \mathscr{G}_{\nu(2)+1} \circ \mathscr{G}_{\nu(1)} \text {. }
$$

By Lemma (2.7) $\mathscr{H}$ is a growth function for $f$. If $\mathscr{H}(i) \neq 0$ then the following system of inequalities must hold.

$$
\begin{aligned}
\nu(1) & \leq i, \\
\nu(2)+1 & \leq \mathscr{G}_{\nu(1)}(i), \\
\nu(3)+1 & \leq \mathscr{G}_{\nu(2)+1} \circ \mathscr{G}_{\nu(1)}, \\
\nu(4)+1 & \leq \mathscr{G}_{\nu(3)+1} \circ \mathscr{G}_{\nu(2)+1} \circ \mathscr{G}_{\nu(1)}, \\
\ldots & \ldots \\
& \ldots \\
\nu(n)+1 & \leq \mathscr{G}_{\nu(n-1)+1} \circ \mathscr{G}_{\nu(n-2)+1} \circ \ldots \circ \mathscr{G}_{\nu(2)+1} \circ \mathscr{G}_{\nu(1)} .
\end{aligned}
$$

Since $\mathscr{G}_{\nu} \leq \mathscr{G}$ for all $\nu \geq 0$ we also have

$$
\begin{aligned}
\nu(1) & \leq i, \\
\nu(2)+1 & \leq \mathscr{G}(i), \\
\nu(3)+1 & \leq \mathscr{G}^{(2)}(i), \\
\cdots & \cdots \\
\cdots & \ldots \\
\nu(n)+1 & \leq \mathscr{G}^{(n-1)}(i),
\end{aligned}
$$

and therefore

$$
\sum_{j=1}^{n} \nu(j) \leq N-n+1
$$


Thus if (2.9) holds then $\mathscr{H}(i)=0$. Since $\mathscr{H}$ is a growth function for $f$ the inequality (2.9) implies that $f$ vanishes on $\beta_{i} \times \beta$.

\section{CONVERGENCE OF POWER SERIES INVOLVING MATRICES OVER ARTIN LOCAL $k$-ALGEBRAS}

Using the results of the previous section, we will now prove some results on the convegence of formal power series when the variables are replaced by matrices with entries in a local $k$-algebra.

Let $A$ be a topological $k$-algebra such that the set $M_{\beta}^{c}(A)$ is a $k$-algebra. If $A$ is a local $k$-algebra with maximal ideal $\mathscr{M}_{A}$ and residue field $k$ then we have $M_{\beta}^{c}(A)=M_{\beta}^{c}(k) \oplus M_{\beta}^{c}\left(\mathscr{M}_{A}\right)$ as vector spaces over $k$. We can therefore write every matrix $f$ in $M_{\beta}^{c}(A)$ uniquely as the sum of a matrix in $M_{\beta}^{c}(k)$, called the residual part of $f$, and a matrix in $M_{\beta}^{c}\left(\mathscr{M}_{A}\right)$ called the ideal part of $f$. We shall denote the residual part of $f$ by $\operatorname{Re}(f)$ and the ideal part of $f$ by $\operatorname{Idl}(f)$. The algebra $M_{\beta}^{c}(A)$ forms a monoid under matrix multiplication. When we wish to consider that monoid we will denote it by $\left(M_{\beta}^{c}(A), *\right)$.

(3.1) Definition. Let $\mathscr{Z}$ be a finite set and let $\Gamma$ be the free monoid generated by $\mathscr{X}$. A homomorphism $\Theta: \Gamma \rightarrow M_{\beta}^{c}(A)$ is said to be admissible if for every $x$ in $\mathscr{Z}$ we have

$$
\operatorname{ord}^{k}(\operatorname{Re}(\Theta(x)))>0 .
$$

(3.2) Lemma. Let $A$ be a local k-algebra whose maximal ideal $\mathscr{M}_{A}$ is nilpotent. Let $\Theta: \Gamma \rightarrow\left(M_{\beta}^{c}(A), *\right)$ be an admissible homomorphism, where as usual * denotes matrix multiplication. Let $P$ be a formal power series. Then for every $\left(b_{1}, b_{2}\right)$ in $\beta \times \beta$ the series

$$
\sum_{w \in \Gamma} P(w) \cdot \Theta(w)\left(b_{1}, b_{2}\right)
$$

has only finitely many nonzero terms and therefore the series

$$
\sum_{w \in \Gamma} P(w) \Theta(w)
$$

defines a matrix which we denote by $\Theta(P)$. Furthermore, the matrix $\Theta(P)$ belongs to $M_{\beta}^{c}(A)$.

Proof. Let $d$ be a nonnegative integer such that $\mathscr{M}_{A}^{d}=0$. Let $\mathscr{G}: \omega \rightarrow \omega$ be an increasing function and assume that $\mathscr{G}$ is a growth function for $\Theta(x)$ for every element $x$ of $\mathscr{X}$. It is easy to see that there is such a function $\mathscr{G}$. Furthermore we may assume that $\mathscr{G}$ is also a growth function for the identity matrix $\delta$. If $w=x_{1} \cdot x_{2} \cdots \cdots x_{L}$ is an element of $\Gamma$ of length $L$, with each $x_{j}$ in $\mathscr{X}$ then for $1 \leq j \leq L$ we have $\Theta\left(x_{j}\right)=\operatorname{Re}\left(\Theta\left(x_{j}\right)\right)+\operatorname{Idl}\left(\Theta\left(x_{j}\right)\right)$. Therefore $\Theta(w)$ is a sum of finitely many terms of the form $f=g_{1} * g_{2} * \cdots * g_{L}$ where for $1 \leq j \leq L$ we have either $g_{j}=\operatorname{Re}\left(\Theta\left(x_{j}\right)\right)$ or $g_{j}=\operatorname{Idl}\left(\Theta\left(x_{j}\right)\right)$. For each such term $f$, let

$$
S_{f}=\left\{j \mid 1 \leq j \leq L \text { and } g_{j}=\operatorname{Idl}\left(\Theta\left(x_{j}\right)\right)\right\} .
$$

If $S_{f}$ has $\geq d$ elements then $f=0$. Suppose that $f \neq 0$. Enumerate $S_{f}$ as a sequence $j(1)<j(2)<\cdots<j(n-1)$, where we adopt the convention that 
$n=1$ if $S_{f}$ is empty. We also let $j(0)=0$. For $1 \leq k \leq n$ let $f_{k}$ be defined by

$$
f_{k}= \begin{cases}\mathscr{G}_{j(k-1)+1} * \mathscr{G}_{j(k-1)+2} * \cdots * \mathscr{G}_{j(k)} & \text { if } 1 \leq k \leq n-1, \\ \mathscr{G}_{j(n-1)+1} * \mathscr{G}_{j(n-1)+2} * \cdots * \mathscr{G}_{L} & \text { if } k=n .\end{cases}
$$

Furthermore, $\mathscr{G}_{\nu(k)}$ is a growth function for $f_{k}$ for $1 \leq k \leq n$ where $\nu(k)$ is defined by

$$
\nu(k)= \begin{cases}j(k)-j(k-1)-1 & \text { if } k<n, \\ L-j(n-1) & \text { if } k=n .\end{cases}
$$

Therefore, $\sum_{k=1}^{n} \nu(k)=L-n+1$.

Let $b_{1}$ be an element of $\beta_{i}$ and let $N=\sum_{j=0}^{d-1} \mathscr{G}^{(j)}(i)$. Since $f \neq 0$ we must have $n-1<d$. Therefore by Lemma (2.8), if $L>N$ then $f$ vanishes on $\beta_{i} \times \beta$. Thus if $w$ is a word of length greater than $N$ then $\theta(w)\left(b_{1}, b_{2}\right)=0$. This proves that (3.3) is a finite sum and that the series (3.4) defines a matrix, which we shall denote by $\Theta(P)$. It remains to prove that $\Theta(P)$ belongs to $M_{\beta}^{c}(A)$. Let $i \geq 0$. We already showed that $\Theta(w)$ vanishes on $\beta_{i} \times \beta$ for every $w$ in $\Gamma$ of length greater than $N$. On the other hand, if $w$ has length $\leq N$ then since $\Theta(w)$ belongs to $M_{\beta}^{c}(A)$ we can find an integer $z(w, i)$ such that $\Theta(w)$ vanishes on $\beta_{i} \times \beta_{j}$ for all $j \geq z(w, i)$. Since there are only finitely many $w$ in $\Gamma$ of length $\leq N$, we can find an integer $z(i)$ such that $\Theta(w)$ vanishes on $\beta_{i} \times \beta_{j}$ for all $j \geq z(i)$ and for all $w$ in $\Gamma$. Therefore $\Theta(P)$ vanishes on $\beta_{i} \times \beta_{j}$ for all $j \geq z(i)$, which proves that $\Theta(P)$ is in $M_{\beta}^{c}(A)$.

\section{The Universal Deformation Rings $\bar{A}$}

In this section we introduce the basic object of study in this paper, namely the $k$-algebra $\bar{A}$. In the next section, we will relate the $k$-algebra $\bar{A}$ and certain of its quotients to formal moduli problems for modules over local $k$-algebras.

If $S$ is a set then a function $f$ from the free commutative monoid generated by $S$ to a commutative $k$-algebra $A$ is called a formal power series in commuting variables in $S$ with coefficients in $A$. The set of all such functions is an $A$-algebra under addition, convolution, and scalar multiplication. If the set $S$ is infinite then there is another more restrictive notion of formal power series with coefficients in $S$ : we will say that a function $f$ from the free commutative monoid generated by $S$ to $A$ is a formal power series in the narrow sense in commuting variables in $S$ with coefficients in $A$ if for every positive integer $n$ there are only finitely many words of length $n$ in the support of $f$. One discusses algebras of formal power series in noncommuting variables in these two senses by using the free monoid instead of the free commutative monoid. The algebras of formal power series in either sense are complete linearly topologized $k$-algebras but in general the topology on the algebra is only defined by the maximal ideal in the case of the algebras of formal power series in the narrow sense. We introduce these concepts so that we can discuss the $k$-algebra $\bar{A}$ without confusion. In general, the $k$-algebra $\bar{A}$ is an algebra of power series in commuting variables in neither sense, as we will now explain.

Let $\mathscr{Y}=\beta \times \beta \times W$ where $W=\{1,2, \ldots, n\}$ and let $k[\mathscr{Y}]$ denote the polynomial ring over $k$ whose indeterminates are the elements of $\mathscr{Y}$. For $1 \leq i \leq n$ let $\widehat{X}_{j}$ denote the $\beta \times \beta$ matrix with values in the set $\mathscr{Y}$ given by 
$\widehat{X}_{i}\left(b_{1}, b_{2}\right)=\left(b_{1}, b_{2}, i\right)$. If $S$ is a subset of $\mathscr{Y}$ we may identify $S$ with an $n$-tuple $S_{1}, \ldots, S_{n}$ of $n \times n$ matrices with entries in $k[\mathscr{Y}]$ by the rule

$$
S_{i}\left(b_{1}, b_{2}\right)= \begin{cases}\left(b_{1}, b_{2}, i\right) & \text { if }\left(b_{1}, b_{2}, i\right) \in \mathscr{Y}, \\ 0 & \text { if }\left(b_{1}, b_{2}, i\right) \notin \mathscr{Y} .\end{cases}
$$

Denote by $D$ the set of all subsets $S$ of $\mathscr{Y}$ such that the associated matrices $S_{1}, \ldots, S_{n}$ all belong to $M_{\beta}^{c}(k[\mathscr{Y}])$, where for the purposes of this definition $k[\mathscr{Y}]$ is given the discrete topology. If $S$ is a subset of $\mathscr{Y}$ belonging to $D$, denote by $\bar{A}_{s}$ the topological $k$-algebra of formal power series in the narrow sense in commuting variables in $S$ with coefficients in $k$. If $S$ and $T$ belong to $D$ and if $S$ is a subset of $T$ then we have a surjective homomorphism $f_{T, S}: \bar{A}_{T} \rightarrow \bar{A}_{S}$ whose values on the set $T$ of generators of $\bar{A}_{T}$ is given by

$$
f_{S, T}\left(b_{1}, b_{2}, i\right)=S_{i}\left(b_{1}, b_{2}\right),
$$

for all $\left(b_{1}, b_{2}, i\right)$ in $T$. Denote by $\bar{A}$ the topological $k$-algebra which is the inverse limit of the algebras $\bar{A}_{S}$ with $S$ in $D$. Although $\bar{A}$ is a complete linearly topologized $k$-algebra, we must emphasize that the topology on $\bar{A}$ is not the topology defined by the maximal ideal of $\bar{A}$ if $\mathscr{Y}$ is infinite. Our goal in this section is to investigate some of the properties of $\bar{A}$.

We begin with the simple remark that there is a natural embedding of $k[\mathscr{Y}]$ into $\bar{A}$ with dense image and that the matrices $\widehat{X}_{1}, \ldots, \widehat{X}_{n}$ may be regarded as $\beta \times \beta$ matrices with entries in $\bar{A}$. We note that $\bar{A}$ is a completion of $k[\mathscr{Y}]$ whose elements may be regarded as formal power series in the elements of $\mathscr{Y}$. However, we should point out that in these series there may be infinitely many terms of a given degree. For example, the series $\sum_{c \in \beta} \widehat{X}_{i}\left(b_{1}, c\right) \widehat{X}_{i}\left(c, b_{2}\right)$ belongs to $\bar{A}$ for all $\left(b_{1}, b_{2}, i\right)$ in $\mathscr{Y}$ and has infinitely many terms of degree 2 if $\mathscr{Y}$ is infinite. Thus $\bar{A}$ is not an algebra of formal power series in the narrow sense if $\mathscr{Y}$ is infinite. On the other hand, even if $\mathscr{Y}$ is infinite, the ring $\bar{A}$ does not contain the formal power series $\sum_{c \in \beta} \widehat{X}_{i}(c, c)$. So $\bar{A}$ is not an algebra of formal power series in the wider sense either. In order to emphasize the similarity of $\bar{A}$ to a power series ring and at the same time to draw a necessary distinction, we will also denote $\bar{A}$ by $k\left[\left[\left[\widehat{X}_{1}, \ldots, \widehat{X}_{n}\right]\right]\right]$.

(4.1) Lemma. The matrices $\widehat{X}_{1}, \ldots, \widehat{X}_{n}$ belong to $M_{\beta}^{c}(\bar{A})$. Furthermore, suppose that $C_{1}, \ldots, C_{n}$ are $\beta \times \beta$ matrices with values in $k$ such that $\operatorname{ord}^{k}\left(C_{i}\right)>$ 0 for $1 \leq i \leq n$. Let $\mathscr{Z}=\left\{x_{1}, \ldots, x_{n}\right\}$ be a set of $n$ indeterminates, $\Gamma$ the free monoid generated by $\mathscr{X}$ and $\Theta: \Gamma \rightarrow\left(M_{\beta}^{c}(\bar{A})\right.$, *) the unique homomorphism such that $\boldsymbol{\Theta}\left(x_{i}\right)=C_{i}+\hat{X}_{i}$. If $P: \Gamma \rightarrow \bar{A}$ is a power series in noncommuting variables in $\mathscr{X}$ with coefficients in $\bar{A}$ then for every element $\left(b_{1}, b_{2}\right)$ of $\beta \times \beta$ the series

$$
\sum_{w \in \Gamma} P(w) \cdot \boldsymbol{\Theta}(w)\left(b_{1}, b_{2}\right)
$$

converges in $\bar{A}$. Consequently the series

$$
\sum_{w \in \Gamma} P(w) \cdot \boldsymbol{\Theta}(w)
$$


defines a matrix with entries in $\bar{A}$ which we denote by $\Theta(P)$. Furthermore, the matrix $\boldsymbol{\Theta}(P)$ belongs to $M_{\beta}^{c}(\bar{A})$.

Proof. The first assertion is quite easy and is left to the reader. For every $S$ in $D$ and every nonnegative integer $d$ let

$$
\bar{A}_{S, d}=\bar{A}_{S} / \mathscr{M}_{S}^{d}
$$

where $\mathscr{M}_{S}$ denotes the maximal ideal of $\bar{A}_{S}$. The topology on $\bar{A}_{S}$ is defined by the maximal ideal $\mathscr{M}_{S}$ and the topology defined on $\bar{A}_{S, d}$ by its maximal ideal is discrete. We have a natural continuous homomorphism of $\bar{A}$ onto $\bar{A}_{S}$ and therefore onto $\bar{A}_{S, d}$. The series (4.2) in $\bar{A}$ corresponds term by term to a series in $\bar{A}_{S, d}$ which converges, by Lemma 3.2 , to the $\left(b_{1}, b_{2}\right)$ th entry of a matrix $\boldsymbol{\Theta}(P ; S, d)$ in $M_{\beta}^{c}\left(\bar{A}_{S, d}\right)$. It is clear that the natural mapping of $\bar{A}_{S, d+1}$ onto $\bar{A}_{S, d}$ maps $\Theta(P ; S, d+1)$ onto $\Theta(P ; S, d)$. Therefore, for every $S$ in $D$ the series (4.3) in $M_{\beta}^{c}(\bar{A})$ corresponds term by term to a series in $M_{\beta}^{c}\left(\bar{A}_{S}\right)$ which converges entry by entry to a matrix $\Theta(P ; S)$. If $S$ and $T$ are elements of $D$ such that $S$ is a subset of $T$, the natural mapping of $\bar{A}_{T}$ onto $\bar{A}_{S}$ maps $\Theta(P ; T)$ entry by entry onto $\Theta(P ; S)$. It follows that the series (4.3) determines a matrix $\Theta(P)$ in $M_{\beta}^{c}(\bar{A})$ which is the sum of the series.

(4.4) Lemma. Let $A$ be a local $k$-algebra with residue field $k$. Suppose that the maximal ideal of $A$ is nilpotent and let $A$ have the discrete topology. Let $\mathscr{X}=\left\{x_{1}, \ldots, x_{n}\right\}$ be a set of $n$ indeterminates, let $\Gamma$ be the free monoid on $\mathscr{X}$ and let $\boldsymbol{\Theta}_{0}: \Gamma \rightarrow\left(M_{\beta}^{c}(A), *\right)$ be an admissible homomorphism. For $1 \leq i \leq n$ let $C_{i}$ be the residual part of $\Theta_{0}\left(x_{i}\right)$. Let $\Theta: \Gamma \rightarrow\left(M_{\beta}^{c}(\bar{A})\right.$, *) be the unique homomorphism such that $\Theta\left(x_{i}\right)=C_{i}+\widehat{X}_{i}$ for $1 \leq i \leq n$. Then there is one and only one continuous $k$-algebra homomorphism $\theta: \bar{A} \rightarrow A$ such that for all $w$ in $\Gamma$ and for all $\left(b_{1}, b_{2}\right)$ in $\beta \times \beta$ we have

$$
\theta\left(\boldsymbol{\Theta}(w)\left(b_{1}, b_{2}\right)\right)=\Theta_{0}(w)\left(b_{1}, b_{2}\right) .
$$

Proof. Since $k[\mathscr{Y}]$ is dense in $\bar{A}$, a continuous $k$-algebra homomorphism from $\bar{A}$ to $A$ is determined by its values on $k[\mathscr{Y}]$. By definition of $\mathscr{Y}$ and of the matrices $\widehat{X}_{i}$, a $k$-algebra homomorphism from $\bar{A}$ to $A$ is therefore determined by its values on elements of the form $\Theta\left(x_{i}\right)\left(b_{1}, b_{2}\right)$ with $\left(b_{1}, b_{2}, i\right)$ in $\mathscr{Y}$. Therefore there is at most one homomorphism $\theta: \bar{A} \rightarrow A$ satisfying (4.5) for all $\left(b_{1}, b_{2}\right)$ in $\beta \times \beta$ and all $w$ in $\Gamma$. It remains to show that such a homomorphism exists. For $1 \leq i \leq n$ let $S_{i}$ denote the set of all $\left(b_{1}, b_{2}\right)$ in $\beta \times \beta$ such that the ideal part $\operatorname{Idl}\left(\Theta_{0}\left(x_{i}\right)\left(b_{1}, b_{2}\right)\right)$ of $\Theta_{0}\left(x_{i}\right)\left(b_{1}, b_{2}\right)$ is nonzero. Then $S_{i}$ belongs to $D$ for each $i$ and therefore the union $S=\bigcup_{i=1}^{n} S_{i}$ of the $S_{i}$ belongs to $D$. If $\theta_{S}: S \times W \rightarrow A$ is the function defined by

$$
\theta_{S}\left(b_{1}, b_{2}, i\right)=\operatorname{Idl}\left(\Theta_{0}\left(x_{i}\right)\left(b_{1}, b_{2}\right)\right)
$$

for all $\left(b_{1}, b_{2}, n\right)$ in $S \times W$ then $\theta_{S}$ determines a $k$-algebra homomorphism from $k[S]$ to $A$, and we also denote this homomorphism by $\theta_{S}$. Let $d$ be a positive integer such that $\mathscr{M}^{d}=0$. Clearly, $\theta_{S}$ vanishes on $\mathscr{M}_{X}^{d} \cap k[S]$ and is therefore continuous with respect to the $\mathscr{M}_{S}$-adic topology on $k[S]$. Therefore $\theta_{S}$ extends to a continuous homomorphism, which we also denote by $\theta_{S}$, from 
$\bar{A}_{S}$ into $A$. Let $\theta: \bar{A} \rightarrow A$ be the composition of $\theta_{S}: \bar{A}_{S} \rightarrow A$ with the natural homomorphism of $\bar{A}$ onto $\bar{A}_{S}$. Then by construction we have

$$
\theta\left(\Theta\left(x_{i}\right)\left(b_{1}, b_{2}\right)\right)=\Theta_{0}\left(x_{i}\right)\left(b_{1}, b_{2}\right)
$$

for all $\left(b_{1}, b_{2}, i\right)$ in $\mathscr{Y}$. Since $\Theta$ and $\Theta_{0}$ are both homomorphisms, the set of all $w$ in $\Gamma$ such that $(4.5)$ holds for all $\left(b_{1}, b_{2}\right)$ in $\beta \times \beta$ is a submonoid of $\Gamma$ containing $x_{1}, \ldots, x_{n}$ and is therefore $\Gamma$ itself.

We describe the moduli problem of which the $k$-algebra $\bar{A}$ is the solution. Let $\mathscr{Z}=\left\{x_{1}, \ldots, x_{n}\right\}$ be a finite set and let $\Gamma$ be the free monoid generated by $\mathscr{X}$. Let $\beta=\bigvee_{i=0}^{\infty} \beta_{i}$ and let $\Theta_{k}: \Gamma \rightarrow M_{\beta}^{c}(k)$ be an admissible representation. Denote by $\mathscr{A L}_{k}$ the category of artin local $k$-algebras with residue class field $k$ and by $\mathscr{E} n s$ the category of sets. Let

$$
\mathscr{F}: \mathscr{A} \mathscr{L}_{k} \rightarrow \mathscr{E} n s
$$

be the functor which associates to each artin local $k$-algebra $A$ the set $\mathscr{F}(A)$ of all admissible homomorphisms $\Theta_{0}: \Gamma \rightarrow M_{\beta}^{c}(A)$ such that $\operatorname{Re}\left(\Theta_{0}\right)=\Theta_{k}$.

(4.6) Theorem. The functor $\mathscr{F}: \mathscr{A L}_{k} \rightarrow \mathscr{E} n s$ is prorepresentable. In fact, for every artin local $k$-algebra $A$ with residue class field $k$ there is a natural bijection from the set $F(A)$ to the set $\operatorname{Hom}_{\text {cont }}(\bar{A}, A)$ of continuous $k$-algebra homomorphisms from $\bar{A}$ to $A$.

Proof. This follows at once from Lemma 4.4.

\section{FORMAL MODULI OF MODULES}

Let the notation and assumptions be as in Theorem (4.6). In particular, $k$ denotes any field; $\mathscr{X}=\left\{x_{1}, \ldots, x_{n}\right\}$ is a finite set of indeterminates and $\Gamma$ is the free monoid generated by $\mathscr{X} ; \boldsymbol{\Theta}_{k}: \Gamma \rightarrow M_{\beta}^{c}(k)$ is an admissible representation in the sense of Definition (2.10), where Re denotes the residual part as defined at the beginning of $\S 3 ; \mathscr{A L}_{k}$ denotes the category of artin local $k$-algebras; $\mathscr{E} n s$ denotes the category of sets, $\mathscr{F}$ denotes the functor from $\mathscr{A L}_{k}$ to $\mathscr{E} n s$ which associates to each artin local $k$-algebra $A$ the set $\mathscr{F}(A)$ of all admissible (in the sense of Definition (3.1)) homomorphisms $\Theta_{0}: \Gamma \rightarrow M_{\beta}^{c}(A)$ such that $\operatorname{Re}\left(\boldsymbol{\Theta}_{0}\right)=\boldsymbol{\Theta}_{k}$. Let $k^{\Gamma}$ denote the set of all functions from $\Gamma$ to $k$. According to our definitions and conventions in $\S 4, k^{\Gamma}$ is the $k$-algebra of formal power series in noncommuting variables in $\mathscr{X}=\left\{x_{1}, \ldots, x_{n}\right\}$ with coefficients in $k$.

The $k$-algebra $\bar{A}$ was defined at the beginning of $\S 4$. Let $\Theta: \Gamma \rightarrow\left(M_{\beta}^{c}(\bar{A}), *\right)$ be the unique monoid homomorphism such that

$$
\boldsymbol{\Theta}\left(x_{i}\right)=\boldsymbol{\Theta}_{k}\left(x_{i}\right)+\widehat{X}_{i}
$$

for $1 \leq i \leq n$. For every formal power series $P: \Gamma \rightarrow k$ in $k^{\Gamma}$ the matrix $\Theta(P)$ is well defined, by Lemma (4.1). Therefore we can regard $\Theta$ as a $k$-algebra homomorphism from $k^{\Gamma}$ to $M_{\beta}^{c}(\bar{A})$. Let $J$ be a closed two-sided ideal in $k^{\Gamma}$. Let $\mathscr{I}$ denote the closed ideal in $\bar{A}$ generated by the entries of the matrices $\Theta(P)$ with $P$ in $J$. 
(5.1) Lemma. Let $\boldsymbol{\Theta}, J$, and $\mathcal{I}$ be as above. Let $A$ be an artin local $k$ algebra with residue field $k$ and let $\Theta_{0}: k^{\Gamma} \rightarrow M_{\beta}^{c}(A)$ be an admissible homomorphism such that $\operatorname{Re}\left(\Theta_{0}\right)=\Theta_{k}$. Then $\Theta_{0}$ is trivial on $J$ if and only if the homomorphism $\theta: \bar{A} \rightarrow A$ corresponding to $\Theta_{0}$ by Theorem (4.6) is trivial on $\mathscr{I}$.

Proof. Denote by $M_{\beta}^{c}(\theta)$ the $k$-algebra homomorphism from $M_{\beta}^{c}(\bar{A})$ to $M_{\beta}^{c}(A)$ induced by $\theta$. Then we have $\Theta_{0}=M_{\beta}^{c}(\theta) \circ \Theta$. Therefore a formal power series $P$ in $k^{\Gamma}$ is in the kernel of $\Theta_{0}$ if and only if every entry of $\Theta(P)$ lies in the kernel of $\theta$. In particular, $\theta_{0}$ is trivial on $J$ if and only if $\theta$ is trivial on $\mathscr{I}$.

(5.2) Theorem. Let the notation and assumptions be as in Lemma (5.1). Let $\mathscr{R}=k^{\Gamma} / J$ and let $\mathscr{M}_{\mathscr{R}}$ be the maximal ideal of $\mathscr{R}$. Let $\mathscr{F}_{\mathscr{R}}: \mathscr{A L}_{k} \rightarrow \mathscr{E} n s$ be the functor which associates to each artin local $k$-algebra $A$ with residue class field the set $\mathscr{F}_{\mathscr{R}}(A)$ of all k-algebra homomorphisms $\rho: \mathscr{R} \rightarrow M_{\beta}^{c}(A)$ such that $\operatorname{ord}^{k}(r)>0$ for all $r$ in $\mathscr{M}_{\mathscr{R}}$. Let $\xi$ belong to $\mathscr{F}_{\mathscr{R}}(k)$ and let $\mathscr{F}_{\mathscr{R}}^{\xi}: \mathscr{A L}_{k} \rightarrow \mathscr{E}_{n s}$ be the subfunctor of $\mathscr{F}_{\mathscr{R}}$ which associates to each artin local $k$-algebra $A$ the set $F_{\mathscr{R}}^{\xi}(A)$ of all $\rho$ in $\mathscr{F}_{\mathscr{R}}(A)$ such that the residual part $\operatorname{Re}(\xi)$ of $\rho$ is equal to $\xi$. Then $\mathscr{F}_{\mathscr{R}}^{\xi}$ is a prorepresentable functor. In fact, for any artin local $k$-algebra $A$ with residue class field $k$ there is a natural bijection from the set $\mathscr{F}_{\mathscr{R}}^{\xi}(A)$ to the set Hom $_{\text {cont }}(\bar{A} / \mathscr{I}, A)$ of continuous $k$-algebra homomorphisms from $\bar{A} / \mathscr{I}$ to A.

Proof. This follows at once from Lemma (5.1) and Theorem (4.6).

(5.3) Lemma. Let $\mathscr{R}$ be as in Theorem (5.2) and let $M$ be an $\mathscr{R}$-module which is complete and separated with respect to the $\mathscr{M}_{\mathscr{R}}$-adic topology on $M$. For every nonnegative integer $i$ let $\beta_{i}$ be a basis for the $k$-vector space $\mathscr{M}_{\mathscr{R}}^{i} M / \mathscr{M}_{\mathscr{R}}^{i+1} M$, where we adopt the convention that $\mathscr{M}_{\mathscr{R}}^{i}=\mathscr{R}$ for $i=0$. Let $\beta=\bigvee_{i=0}^{\infty} \beta_{i}$ be the union of the sets $\beta_{i}$. Then $M$ is isomorphic as a topological $k$-module to the free topological $k$-module with Schauder basis $\beta$.

Proof. This is quite straightforward and is left to the reader.

(5.4) Lemma. Let $A$ be an artin local $k$-algebra with residue class field $k$ and let $M$ be a topological $\mathscr{R} \otimes A$ module which is flat over $A$ and which is complete and separated with respect to the $\mathscr{M}_{\mathscr{R}}$-adic topology on $M$. Then there is a set $\beta=\bigvee_{i=0}^{\infty} \beta_{i}$ such that $M$ is isomorphic as a topological $A$ module to $\mathscr{S}(A ; \beta)$. Proof. Since $A$ is an artin local $k$-algebra, we can find a descending chain of ideals

$$
0=I_{0} \subset I_{1} \subset \cdots \subset I_{n}=\mathscr{M}_{A}
$$

such that $I_{\nu} / I_{\nu-1}$ is isomorphic to the $A$-module $k$ for $1 \leq \nu \leq n$. Let $\alpha_{1}, \ldots, \alpha_{n+1}=1$ be a basis for $A$ over $k$ and such that $\alpha_{1}, \ldots, \alpha_{\nu}$ is a basis for $I_{\nu}$ for $1 \leq \nu \leq n$. For $0 \leq \nu \leq n$ let $M_{\nu}$ denote the submodule $I_{\nu} M$ of $M$ and let $M_{n+1}=M$. Since $M$ is $A$-flat, multiplication by $\alpha_{\nu}$ induces a topological isomorphism $\hat{\alpha}_{\nu}$ of $M / \mathscr{M}_{A} M$ onto $M_{\nu} / M_{\nu-1}$ for $1 \leq \nu \leq n+1$. Therefore $M$ is topologically isomorphic as a topological vector space over $k$ to the direct sum of the spaces $M_{\nu} / M_{\nu-1}$ for $1 \leq \nu \leq n+1$. We can make this direct sum into an $A$-module by identifying it with $\bar{A} \otimes_{k}\left(M / \mathscr{M}_{A}\right)$ by means of the isomorphisms $\hat{\alpha}_{\nu}: M / \mathscr{M}_{A} \rightarrow M_{\nu} / M_{\nu-1}$ for $1 \leq \nu \leq n+1$. Since $M / \mathscr{M}_{A}$ 
satisfies the hypothesis of Lemma (5.3), it is isomorphic to $\mathscr{S}(k ; \beta)$. It follows that $M$ is isomorphic as a topological $A$-module to $A \otimes_{k} \mathscr{S}(k ; \beta)$ which is isomorphic to the $A$-module $\mathscr{S}(A ; \beta)$.

(5.5) Definition. Let $A$ be an artin local $k$-algebra with residue field $k$. Let $M$ be a topological $A$-module topologically isomorphic to $\mathscr{S}(A ; \beta)$ for some $\beta=\bigvee_{i=0}^{\infty} \beta_{i}$. By a Schauder basis for $M$ we mean the preimage of $\beta$ under a topological isomorphism of $M$ onto $\mathscr{S}(A ; \beta)$.

(5.6) Theorem. Let $\mathscr{D}_{(\mathscr{M}, \beta)}: \mathscr{A L}_{k} \rightarrow \mathscr{E}$ ns be the functor which associates to each object $A$ of $\mathscr{A L}_{k}$ the set of isomorphism classes of triples $\left(M_{A}, \beta^{A}, \phi_{A}\right)$ where

(1) $M_{A}$ is an A-flat topological $\mathscr{R} \otimes A$ module;

(2) $\beta^{A}=\bigvee_{i=0}^{\infty} \beta_{i}^{A}$ is a Schauder basis for the A-module $M$;

(3) $\phi_{A}$ is an $\mathscr{R}$-isomorphism of $M_{A} \otimes_{A} k$ onto $M$;

(4) The mapping $m \mapsto \phi_{A}(m \otimes 1)$ from $M_{A}$ to $M$ maps $\beta_{i}^{A}$ bijectively onto $\beta_{i}$ for all $i \geq 0$.

Then $\mathscr{D}$ is prorepresentable. In fact, $\mathscr{D}$ is naturally isomorphic to the functor $A \mapsto \operatorname{Hom}_{\text {cont }}(\bar{A} / \mathscr{I}, A)$.

Proof. Using the Schauder basis $\beta$ we can identify $M$ with the free topological $k$-module $\mathscr{S}(k ; \beta)$. Since $\mathscr{R}$ acts continuously on $M$ with respect to the $\mathscr{M}_{\mathscr{R}}$-adic topology on $M$, by Proposition (1.2) and by (1.3) we have a $k$-algebra homomorphism $\Theta_{k}: R \rightarrow M_{\beta}^{c}(k)$ such that each element $r$ of $\mathscr{R}$ acts on $M$ via the matrix $\Theta_{k}(r)$. Furthermore, by Lemma (5.3) we have $\operatorname{ord}^{k}\left(\boldsymbol{\Theta}_{k}(r)\right)>0$ for all $r$ in $\mathscr{M}_{\mathscr{R}}$. If $A$ is an artin local $k$-algebra with residue field $k$ and $\left(M_{A}, \beta^{A}, \phi^{A}\right)$ is an element of $\mathscr{D}(A)$ we may, by Lemma (5.4), identify $M_{A}$ with the free topological $A$-module $\mathscr{S}(A ; \beta)$. Since the $A$-module structure on $\mathscr{S}(A ; \beta)$ is evident, the $\mathscr{R} \otimes_{k} A$-module structure on $M_{A}$ is uniquely determined by a $k$-algebra homomorphism $\Theta_{0}: \mathscr{R} \rightarrow M_{\beta}^{c}(A)$. The isomorphism $\phi^{A}: M_{A} \otimes_{A} k \rightarrow M$ can be taken to be the mapping defined by $\phi^{A}(f \otimes 1)(b)=\lambda(f(b))$ for all $b$ in $\beta$, where $\lambda$ denotes the natural mapping of $A$ onto $k$. Condition (3) is equivalent to the condition that $\Theta_{k}$ is the residual part of $\boldsymbol{\Theta}_{0}$. Therefore if we let $\xi=\Theta_{k}$, we see that $\mathscr{D}$ is naturally isomorphic to the functor $F_{\mathscr{R}}^{\xi}$ defined in Theorem (5.2). Our result now follows from Theorem (5.2).

\section{REFERENCES}

[A] Michael Artin, Algebraization of formal moduli I, Global Analysis, A collection of mathematical papers published in honor of K. Kodaira, Univ. of Tokyo Press, Tokyo, 1969.

[A-I-K] A. Altman, A. Iarrobino, and S. Kleiman, Irreducibility of the compactified jacobian, Real and Complex Singularities, Proc. Ninth Nordic Summer School/NAVF Sympos. Math., Oslo, 1976, pp. 1-12.

[B] Nicolas Bourbaki, Algebra, Addison-Wesley, Reading, Mass.

[D] C. D’Souza, Compactification of generalized jacobians, Proc. Indian Nat. Sci. Acad. 88A (1979), 419-457.

[G] Jyotsna Gokhale, Exploring the compactified Picard variety of a singular cure, Ph.D. thesis, Brandeis Univ., 1987.

[G-K] G.-M. Geuel and Horst Knörrer, Einfache Kurvensingularitäten und torsionfreie Moduln, Math. Ann. 270 (1985), 417-425. 
[Gr] Alexander Grothendieck, EGA I, Inst. Hautes Études Sci. Publ. Math. 4 (1960).

[M] Barry Mazur, Deforming Galois representations, preprint.

[Sch] Michael Schlessinger, Functors of Artin rings, Trans. Amer. Math. Soc. 130 (1968), 208-222.

[Ser] Jean-Pierre Serre, Cohomologie Galoisienne, Lecture Notes in Math., vol. 5, SpringerVerlag, Berlin and New York, 1965.

[Sesh] C. S. Seshadri, Fibres vectoriels sur les courbes algebriques, Astérisque 96 (1982).

[Sh] Pradeep Shukla, Deformations of certain torsion-free sheaves over a curve with planar singularities, Ph.D. thesis, Brandeis Univ., 1987.

[S-O] C. S. Seshadri and T. Oda, Compactifications of the generalized jacobian variety, Trans. Amer. Math. Soc. 253 (1979), 1-90.

Current address, A. Adler: 36 Rolens Drive, Apt. C4, Kingstown, Rhode Island 02881

E-mail address, A. Adler: ara@altdorf.ai.mit.edu

Department of Mathematics, Suffolk University, Boston, Massachusetts 02114 